\title{
Percepción de distancia lingüística en la adquisición de la lengua portuguesa por hispanohablantes
}

\author{
Ana María Díaz Ferrero \\ Universidad de Granada \\ anadiaz@ugr.es
}

\section{Resumen}

Este trabajo tiene por objeto analizar la actitud de los estudiantes hispanohablantes hacia el aprendizaje de la lengua portuguesa en el marco de formación del Grado de Traducción e Interpretación de la Universidad de Granada. Para ello hemos entrevistado a los 43 estudiantes matriculados en el Grado en las asignaturas de Lengua $\mathrm{C}$ portugués en la modalidad presencial divididos en tres niveles de aprendizaje. El resultado de las encuestas que los alumnos han realizado demuestra que tienen una percepción distorsionada de la distancia linguística entre la lengua portuguesa y la lengua española lo cual puede favorecer la aparición de transferencias negativas en el proceso de traducción. Esta percepción de distancia lingüística es mínima en la fase inicial de aprendizaje y crece a medida que aumenta la competencia lingüística del estudiante. Consideramos que estos y otros factores afectivos se deben tener en cuenta a la hora de diseñar una metodología específica para la enseñanza del portugués a hispanohablantes.

Palabras clave: Distancia lingüística. Portugués LE. Factores afectivos. Transferencia negativa.

\section{Resumo}

Este trabalho tem como objetivo analisar a atitude dos estudantes hispanofalantes face a aprendizagem da língua portuguesa nos estudos de Graduação de Traducción e Interpretación da Universidade de Granada. Para tal, foram entrevistados os 43 estudantes inscritos neste curso nas disciplinas de língua portuguesa na modalidade presencial divididos em três níveis de aprendizagem: inicial, intermediário e avançado. $\mathrm{O}$ resultado das entrevistas realizadas mostrou que os estudantes têm uma percepção deturpada da distância linguística entre o português e o espanhol, o qual pode ser a causa de transferências negativas no processo de tradução. Esta percepção de distância linguística é mínima na fase inicial de aprendizagem e aumenta à medida que cresce a competência linguística do estudante. Consideramos que estes e 
outros fatores afetivos devem ser tidos em consideração no momento de criar uma metodologia específica para o ensino da língua portuguesa para hispanofalantes.

Palavras-chave: Distância linguística. Português LE. Fatores afetivos. Transferência linguística.

\section{Introducción}

La proximidad entre el portugués y el español ha sido objeto de estudio de distintas investigaciones que han analizado las ventajas y desventajas que genera la adquisición de estas lenguas próximas (ALMEIDA FILHO, 1995; ALONSO REY, 2004; CAMARENA, 2011; CAMORLINGA, 1997; NATEL, 2002; ORTIZ ÁLVAREZ, 2012; PONCE DE LEÓN, 2007), y las dificultades y falsas transferencias que puede ocasionar dejarse llevar por la aparente similitud de estas lenguas en un proceso de traducción (DIAS, 1991; SABIO PINILLA, 1992; ELAMANE, 1996; CALVO CAPILLA; RIDD, 2009; FÉRRIZ MARTÍNEZ; SANS CLIMENT, 2010; ALONSO REY, 2012; DÍAZ FOUCES, 2012). La proximidad lingüística de estas lenguas sigue generando entre los estudiantes la idea de que pueden realizar un aprendizaje intuitivo y rápido. Noemí Pérez en un estudio sobre la enseñanza del español en Portugal afirma que "la falsa percepción de que es fácil y de que se entiende sin dificultad provoca la creencia generalizada de que su estudio no es necesario" (PÉREZ, 2008, p. 27). Ya en 1937 en la lección inaugural de la cátedra de Filología Románica, pronunciada en Brasil en la Universidad del Distrito Federal, se observó en relación con el estudio de lenguas románicas en la enseñanza secundaria que el francés era materia obligatoria, y que el italiano y el español no lo eran por considerarse de gran facilidad para los brasileños: "semelhantes à nossa língua, lemo-los e compreendemo-los com relativa facilidade, chegando até a falar com tal ou qual correção" (NASCENTES, 1939, p. 73). Aunque la evolución en la metodología de la enseñanza de lenguas próximas ha sido significativa, todavía hoy el aprendizaje del portugués por parte de hispanohablantes se considera una tarea extremadamente fácil e incluso innecesaria. El discente de esta lengua en el marco de los estudios de Traducción e Interpretación debe 
comprender que el objetivo de su formación no es la comprensión más o menos precisa de un texto, sino alcanzar un nivel superior de competencia lingüística ligada a una buena técnica de separación del portugués y el español que le permita llevar a cabo con calidad la práctica de la traducción y de la interpretación.

La experiencia de más de dos décadas de enseñanza de portugués a hispanohablantes nos ha permitido constatar que la gran afinidad semántica y estructural existente entre el portugués y el español facilita el aprendizaje, y las competencias lingüísticas que el estudiante posee de la lengua materna le sirven de referente para la adquisición de la lengua extranjera. No obstante, es indudable también que la proximidad de estas lenguas es a veces engañosa y que el estudiante puede realizar hipótesis falsas e interpretaciones erróneas que provocan errores de traducción. Además, esta influencia negativa de la lengua materna favorece la fosilización de formas incorrectas que no obstaculizan la comunicación pero generan un estancamiento lingüístico principalmente en los niveles intermedio y avanzado. En este escenario de proximidad linguística los factores afectivos como la actitud del alumno y su percepción de la lengua extranjera influyen considerablemente en el proceso de aprendizaje. El objetivo de este artículo es analizar desde un punto de vista cognitivista la evolución de la actitud del estudiante hispanohablante en el proceso de su formación como traductor e intérprete. Estudiaremos concretamente la percepción de dificultad y la percepción de distancia entre la lengua materna (LM) y la lengua extranjera (LE). No se pretende analizar la distancia real entre el español y el portugués sino la distancia percibida por el alumno y las consecuencias que origina esa percepción de más o menos proximidad en el proceso de traducción.

\section{Marco teórico}

El análisis contrastivo y las investigaciones sobre la influencia entre lenguas y la transferencia de elementos de la LM a la LE se remontan a mediados del siglo XX con los trabajos de C. Fries (1945), Weinreich (1953) y Lado (1957), y han servido de base para posteriores investigaciones sobre el proceso de adquisición de la lengua extranjera, análisis de errores y estrategias de aprendizaje. 
Siguiendo estas teorías, cuando se trasladan con éxito elementos de una lengua a otra el proceso se denomina transferencia positiva, y, por el contrario, cuando los elementos transferidos dan lugar a errores se denomina transferencia negativa o interferencia (cf. ODLIN, 1989; GASS; SELINKER; 1983). Este proceso de transferencia se puede producir en todos los niveles de la lengua: semántico, léxico, morfosintáctico, fonético o pragmático, e intervienen no solo factores lingüísticos sino también psicológicos. En este sentido, Kellerman (1983) desarrolló el concepto de psicotipología, que se refiere a la percepción que tiene el estudiante del grado de proximidad de las lenguas. Según este autor, la percepción que tiene el alumno de la distancia existente entre la LM y la LE favorece o dificulta la adquisición de la lengua extranjera, esto es, la transferencia de elementos y estructuras de una lengua a otra depende de la percepción de proximidad tipológica por parte del alumno. Si las dos lenguas se perciben como próximas, el aprendiz tiende a trasladar estructuras y elementos de una lengua a otra. Los planteamientos del análisis contrastivo y el fenómeno de la transferencia han recibido numerosas críticas, pero aún sigue vigente con sucesivas adaptaciones. Diversos autores (CALVI, 2004; FERNANDES; BRITO, 2006; AINCIBURU, 2008; VILLANUEVA, 2011) han aplicado el concepto de psicotipología de Kellerman al proceso de aprendizaje de lenguas y ponen de manifiesto que la percepción de distancia lingüística por parte del estudiante es un factor influyente en el proceso de transferencia lingüística. Patrícia Rosa Lozado (2007) realizó una investigación para determinar de qué manera la distancia lingüística, tanto real como psicológica, influye en el proceso de adquisición del sistema pronominal de la lengua española por hablantes brasileños. Esta autora observó que los alumnos cuando comienzan a estudiar español casi no perciben distancia entre las dos lenguas; sin embargo, tras un contacto más prolongado con la lengua española, terminan por considerar difícil su aprendizaje. Finalmente concluyó que la percepción por parte de los estudiantes de gran distancia lingüística respecto al uso del sistema pronominal en español y en portugués, sumada a la distancia lingüística real, podía influir de forma negativa en la adquisición de la lengua española. Otros autores como Carmen Férriz Martínez y Carles Sans Climent (2010, p. 58) consideran que la noción de percepción de distancia se puede aplicar no solo al proceso 
de aprendizaje de lenguas, sino que también resulta de gran utilidad para explicar determinadas inadecuaciones de traducción en los que, por la similitud formal entre el español y el portugués, el alumno incurre en errores como falsos sentidos o contrasentidos.

La percepción de distancia condiciona la actitud del estudiante ante la lengua extranjera e influye en el desarrollo de la competencia lingüística. Factores afectivos como la motivación, la ansiedad, la valoración de los propios conocimientos lingüísticos, la distancia percibida entre las lenguas o la actitud del alumno, inciden enormemente en el proceso de adquisición de lengua extranjera (HUFEISEN; MARX, 2007, p. 314, apud VILAR, 2014, p. 74). Según la teoría de autoeficacia de Albert Bandura (1997), las expectativas que tiene el alumno sobre sus capacidades para obtener un objetivo determina su conducta. Si poseen un nivel elevado de autoeficacia se enfrentarán a situaciones difíciles con una actitud positiva y confiada. Nuestra experiencia nos ha permitido constatar que desde las primeras etapas de aprendizaje el estudiante percibe las lenguas como semejantes y muestra una actitud positiva y optimista que se acentúa cuando consigue realizar transferencias positivas y llevar a cabo con éxito diferentes actos de comunicación oral y escrita. Este hecho fomenta la motivación y la autoestima del alumno y facilita el aprendizaje, pero al mismo tiempo disfraza el nivel real de competencia lingüística y favorece la falta de atención y el descuido. Cuando el alumno no tiene un conocimiento profundo de la lengua portuguesa y desestima las diferencias existentes entre la LM y la LE se deja llevar por las semejanzas y aumenta el riesgo de aparición de interferencias y de fosilización. El modo de percibir la distancia lingüística influye considerablemente en la actitud, la confianza y la aparición de conciencia del error, factor determinante que ayuda a desarrollar estrategias para evitar caer en la trampa de las falsas analogías. Este proceso podría esquematizarse del siguiente modo:

Percepción de distancia menor de la real $\Rightarrow$ Excesiva confianza

Excesiva confianza $\Rightarrow$ Bajada de la guardia

Bajada de la guardia $\Rightarrow$ Aparición de interferencias

Los estudios publicados sobre este asunto coinciden en proponer un método propio para la enseñanza de estas lenguas. De este modo ya en 
1983 Lombello, El-Dash y Baleeiro (1983, p. 127) en un artículo sobre la elaboración de material didáctico de portugués para hablantes de español de la Universidade Estadual de Campinas señalaban que "um curso de português para falantes de espanhol deve realmente ser diferente dos cursos para outros estrangeiros". Cuando el alumno hispanohablante inicia el aprendizaje de la lengua portuguesa posee un conocimiento pasivo que facilita la comprensión escrita y agiliza el proceso de adquisición de la lengua. Como afirman Kulikowski y González (1999), en un trabajo sobre la enseñanza del español a brasileños el éxito radica en "establecer la justa medida de esa cercanía $\mathrm{y}$, si existe, sacar provecho de ella sin caer en la falsa transparencia y, sobre todo, sin reducir o empobrecer ambas lenguas" (KULIKOWSKI; GONZÁLEZ, 1999, p. 19).

Esa metodología específica de enseñanza de lenguas afines en el caso de la formación de futuros traductores e intérpretes debe estar orientada a alcanzar una competencia lingüística traductora que permita por un lado la comprensión total del texto de origen y por otro un uso adecuado e idiomático de la lengua meta evitando la transmisión de elementos de una lengua a otra. Augusto Múrias (2014, p. 5), traductor del Parlamento europeo, publicó en cinco artículos de la revista $A$ Folha un corpus de dificultades para la traducción entre las lenguas portuguesa y española. Este autor sugiere que la enseñanza del español a portugueses no debe comenzar desde un nivel inicial absoluto sino utilizando textos desde el inicio del aprendizaje. El alumno debe adquirir una buena competencia lingüística que le permita distinguir las semejanzas y las diferencias de las dos lenguas. Las interferencias en el proceso de traducción se producen de forma inconsciente y una solución, según Calvo Capilla y Ridd (2009, p. 158) es incrementar el control en este proceso, es decir, tomar más conciencia para evitar las interferencias. No se trata de crear una atmósfera pesimista ni de reprimir la espontaneidad del alumno frenando su iniciativa de realizar las transferencias positivas que la cercanía lingüística permite, sino de desarrollar lo que denominamos estrés lingüístico positivo. El estrés es un mecanismo de alerta que permite activar los recursos y conocimientos necesarios para poder reaccionar ante un peligro. Un nivel de estrés excesivo o inadecuado produce reacciones adversas y desencadena efectos negativos como el bloqueo mental; es lo que sucede, por ejemplo, cuando la LE se 
percibe como muy difícil o inalcanzable. Por el contrario, un nivel excesivamente bajo de estrés puede ser también negativo porque provoca apatía y falta de atención para reaccionar ante situaciones de amenaza o peligro. Esta es la situación que genera una actitud excesivamente confiada y optimista hacia el aprendizaje de la lengua portuguesa. Neide González en un artículo sobre la enseñanza del español en Brasil afirmaba que "em muitos casos são as crenças na facilidade e o consequiente menosprezo pela língua os que conduzem à acomodação e ao desinteresse que tanto prejudicam a aprendizagem" (GONZÁLEZ, 1989, p. 88-89). Cuando el estrés se maneja de forma adecuada fomenta la atención, estimula la creatividad y permite resolver los problemas con mayor rapidez y eficiencia. Si consideramos que las falsas analogías - no solo léxicas, sino también estructurales y pragmáticas - suponen un peligro y una amenaza para la traducción, el estrés positivo activa nuestros conocimientos para estar alerta en aquellas situaciones en las que las falsas analogías pueden generar errores. Si el estudiante cambia la percepción de la lengua portuguesa, cambiará también su comportamiento y sabrá cuando puede mantener una actitud relajada y confiada, y cuando tiene que activar los mecanismos de alerta necesarios para evitar transferencias negativas.

\section{Metodología}

Este estudio se llevó a cabo durante el segundo semestre del curso académico 2015-2016. El universo objeto de estudio son los alumnos de lengua materna española que estudian portugués durante los cuatro años del Grado de Traducción e Interpretación de la Universidad de Granada. De los 52 alumnos matriculados en las asignaturas de lengua portuguesa, hemos entrevistado a los 43 estudiantes hispanohablantes inscritos en la modalidad de enseñanza presencial. La muestra está compuesta por un grupo heterogéneo de estudiantes universitarios hispanohablantes con una edad comprendida entre 18 y 24 años que ya conocen, al menos, una lengua extranjera (la primera lengua extranjera del estudiante que en el plan de estudios se denomina lengua $\mathrm{B}$ y que puede ser inglés, francés, alemán o árabe) y estudian la lengua portuguesa como lengua C (segunda o tercera 
lengua extranjera). Dividimos la muestra en tres grupos de estudiantes según el nivel de competencia lingüística. El primer grupo está compuesto por 24 alumnos de la fase inicial de aprendizaje, que en el momento de responder el cuestionario se encontraban cursando la asignatura "Lengua C2 Portugués" y cuyo nivel, según la programación docente de la asignatura, corresponde a A2+/B1 del Marco común europeo de referencia para las lenguas (en adelante MCERL) (CONSEJO, 2002). El número de alumnos entrevistados en este grupo representa el $80 \%$ del total de alumnos matriculados en este nivel inicial. El segundo grupo está formado por 11 estudiantes de la fase intermedia de aprendizaje que se encontraban cursando la asignatura "Lengua C6 Portugués" en el momento de responder el cuestionario y cuyo nivel corresponde a B2 del MCERL. Este grupo representa el 78,5\% del total de alumnos matriculados en este nivel. Y por último, el tercer grupo está compuesto por 8 alumnos de nivel avanzado, que se encontraban cursando el último semestre del Grado de Traducción; son estudiantes que ya han cursado todos los niveles de lengua portuguesa, que poseen un nivel B2+/C1 del MCERL y que han realizado, al menos, tres asignaturas de traducción portugués-español. Este grupo representa al $100 \%$ de los alumnos de esta fase de aprendizaje.

\section{Resultados}

\section{1 Nivel inicial}

Un estudiante hispanohablante que decide estudiar portugués es en realidad un "falso principiante" ya que, debido al parecido entre la LM y la LE, posee una alta capacidad de comprensión lectora de la lengua portuguesa. En un estudio comparativo entre el español y el portugués realizado por Jack L. Ulsh se concluye que más del $85 \%$ del vocabulario de la lengua portuguesa tiene cognados en español (ULSH, 1971 , p. X). Estas semejanzas, que se extienden también a la estructuras gramaticales, hacen que el estudiante posea un conocimiento pasivo y perciba desde el principio un elevado grado de familiaridad con la lengua portuguesa lo cual le permite comprender, incluso sin estudiar la lengua, gran parte del contenido de textos 
escritos. Eunice Henriques (2000) realizó un estudio con 300 estudiantes de lengua española y 300 de lengua portuguesa, que nunca habían estudiado la otra lengua, para analizar el nivel de comprensión del texto escrito en portugués por hablantes de español y viceversa, y pudo comprobar que existe un alto índice de comprensión que oscila entre el $58 \%$ y el $94 \%$ dependiendo del contexto o la semejanza léxicosemántica.

En términos generales, en la fase inicial de formación del estudiante, salvo algunas dificultades relacionadas con la expresión y la comprensión oral, el aprendizaje de la lengua portuguesa por parte de hispanohablantes se desarrolla de forma fluida y rápida. Por tanto, la actitud del discente ante el aprendizaje de la nueva lengua es optimista y su percepción de dificultad es casi nula. El alumno que decide estudiar portugués es consciente de la proximidad de estas lenguas y comienza su aventura lingüística con una actitud extremadamente positiva por considerar que conseguirá dominar la lengua en un breve espacio de tiempo. Esta idea no es del todo errónea. Muchos investigadores (ALMEIDA FILHO, 1995; LEIRIA, 1998; AMADO; ACOSTA, 2000; NATEL 2002; CALVO CAPILLA, 2009); DÍAZ FOUCES 2012; BAERLOCHER; LUCHA, 2014) reflejan esta facilidad y rapidez para aprender estas lenguas en la primera etapa de aprendizaje, dado que, si se diseña una metodología específica, se pueden aprovechar las ventajas de la proximidad del portugués y el español y obtener el máximo rendimiento. En palabras de Díaz Fouces (2012, p.121): "se as estratégias formativas forem as adequadas, essa proximidade estructural será um benefício óbvio, em termos de tempo e de esforços formativos".

Los resultados de los cuestionarios revelan que la percepción por parte del alumno de dificultad y de distancia lingüística entre el portugués y el español es mínima en esta primera etapa. El alumno va descubriendo la estructura, el funcionamiento, la morfología y el vocabulario de la LE y detecta muchas analogías entre ambas lenguas. De este modo comprueba que puede aplicar con éxito sus conocimientos de la lengua materna a la lengua extranjera y realizar un significativo número de transferencias positivas que agilizan el aprendizaje, lo cual resulta un factor determinante a la hora de aumentar su confianza y autoeficacia para aprender este idioma. El $87,5 \%$ de los estudiantes de portugués considera durante el primer año 
de aprendizaje que el portugués es una lengua fácil, si la comparan con su primera lengua extranjera o lengua B. La semejanza y la cercanía estructural y gramatical entre el portugués y el español es una ventaja para el $75 \%$ de los encuestados. La semejanza léxica no es un aspecto tan bien valorado, aunque también aparece como una ventaja para el $58,3 \%$ de los estudiantes. El nivel medio de este primer grupo de alumnos, según el MCERL, es A2 +/ B1 y, aunque todavía no han realizado asignaturas específicas de traducción, la gran mayoría $(75 \%)$ tiene la idea de que traducir del portugués al español es bastante fácil, y un porcentaje también elevado $(70,8 \%)$ considera que después de cuatro años de estudio de lengua portuguesa alcanzará el nivel adecuado que le permitirá ejercer con calidad la profesión de traductor e intérprete. Por otro lado, la mayoría (75\%) cree que las falsas semejanzas solo provocan errores de traducción cuando el nivel de conocimiento lingüístico es inicial o intermedio frente al $25 \%$ que considera que pueden provocar errores de traducción también cuando el nivel de conocimiento lingüístico es elevado. En cuanto a las causas por las que las falsas analogías provocan errores de traducción, el $87,5 \%$ considera que se deben a desconocimiento del léxico, seguido de falta de atención (41,6\%); el contexto textual en el que aparece el falso amigo (37,5\%), el cansancio o estrés del traductor $(29,16 \%)$, la falta de competencia traductora $(25 \%) \mathrm{y}$, por último, la urgencia de la traducción y la falta de revisión $(16,6 \%)$ junto con la escasez de diccionarios y otras fuentes de documentación $(16,6 \%)$.

\section{2 Nivel Intermedio}

A medida que el alumno avanza en el conocimiento de la LE va modificando su percepción de la lengua portuguesa, adopta una actitud reflexiva ante la lengua que estudia, va adquiriendo un conocimiento más profundo y poco a poco se va ampliando su percepción de distancia y de dificultad. Camarena Ortiz, después de realizar un análisis de los errores que tienen los mejicanos cuando estudian portugués en un corpus escrito de 104 redacciones de alumnos de niveles intermedio y avanzado, concluyó que existe "una incidencia muy significativa de errores de interferencia, frente a una menor ocurrencia de errores no relacionados con la lengua materna" (CAMARENA, 2011, p. 79). En este sentido, las investigaciones 
científicas indican que las interferencias entre lenguas son mayores cuando las diferencias entre lenguas son mínimas como es el caso del español y el portugués (cf. OLLER; ZIAHOSSEINY, 1970; ORTIZ ÁLVAREZ, 2012). Como sostiene Carmolinga (1997, p. 658) "a divisória entre o português e o espanhol é muito tênue, tornando-se às vezes imperceptível".

Los resultados de nuestro estudio ponen de manifiesto que los estudiantes van modificando su percepción y su actitud hacia la lengua portuguesa. En esta etapa de aprendizaje el alumno empieza a realizar ejercicios de traducción y descubre falsas analogías y elementos de la lengua que pueden provocar interferencias no solo semánticas sino también prosódicas, ortográficas, ortotipográficas, morfológicas, sintácticas o pragmáticas (CARLUCCI; DÍAZ, 2007). El 72,70\% de los estudiantes de portugués sigue considerando que la lengua portuguesa es una lengua fácil en términos generales, si la comparan con su lengua B. El porcentaje de entrevistados que considera la semejanza y la cercanía estructural y gramatical entre el portugués y el español como una ventaja se mantiene alto $(63,63 \%)$. La semejanza léxica empieza a verse como un problema, dado que tan solo un $45,45 \%$ de los estudiantes entrevistados percibe el parecido del léxico como una ventaja. El número de alumnos que cree que traducir del portugués al español es bastante fácil baja a un 63,63\% y se mantiene el porcentaje de alumnos que cree que después de cuatro años de formación universitaria alcanzará la competencia lingüística necesaria para poder ejercer con calidad la profesión de traductor e intérprete. Por otro lado, aumenta el número de estudiantes que considera que las falsas analogías pueden provocar errores de traducción incluso cuando el nivel de conocimiento lingüístico es elevado (36,36\%). En suma, en la etapa intermedia de aprendizaje el alumno profundiza en el estudio de la lengua, aumenta su competencia linguiística y su actitud ante la lengua extranjera también se modifica. El estudiante va conociendo los peligros de la proximidad lingüística y adopta una actitud más prudente tanto en el uso activo de la lengua portuguesa como en el proceso de traducción, en otras palabras, empieza a tener en consideración las estrategias empleadas en su proceso de aprendizaje. Este hecho hace que vaya aumentando su percepción de dificultad y que sea consciente de la posibilidad y el riesgo de interferencia por lo que adopta una actitud más cautelosa que le servirá de protección frente a las falsas 
analogías de las dos lenguas. Respecto a las causas por las que las falsas analogías provocan errores, el $72,7 \%$ considera que la principal causa es el desconocimiento de léxico, seguido del contexto textual $(54,54 \%)$. El cansancio o estrés del traductor puede ser la causa de errores según el $45,45 \%$, y el mismo porcentaje $(45,45 \%)$ considera que la falta de atención y la urgencia para entregar las traducciones junto con la falta de revisión puede ser un motivo de interferencia o error. La falta de competencia traductora puede ser la causa de errores para el $36,36 \%$, y, por último, la escasez de diccionarios y otras fuentes de documentación es un motivo de interferencia para el 18,8\%.

\section{3 Nivel avanzado}

En la tercera y última fase de formación académica, es decir, en el último curso del Grado de Traducción e Interpretación, el estudiante domina gran parte del vocabulario, de las estructuras gramaticales y de la pragmática de la lengua extranjera. Su contacto progresivo con la traducción del portugués al español le ha permitido adoptar una actitud más crítica y reflexiva y, en el plano pragmático, descubre que es necesario mantener una actitud distante hacia el texto original para no crear un texto meta extraño y desnaturalizado. En esta etapa la competencia lingüística del alumno ha mejorado considerablemente, pero existe un alto riesgo de estancamiento. La mayoría de las investigaciones sobre el aprendizaje del portugués por alumnos hispanohablantes y del español por alumnos lusófonos advierten sobre las dificultades de progresión en la adquisición de la LE en niveles avanzados y sobre el riesgo de fosilización.

As opiniões aduzidas e os dados apresentados demonstram que, a vantagem inicial, quando da aprendizagem de uma língua cognata, nem sempre (aliás, raras vezes) culmina no domínio quase perfeito e rápido da língua visada. Muito pelo contrário, o mais provável é estacionar numa interlíngua, mais ou menos distante da meta. Facilmente a fossilização toma conta, inviabilizando qualquer progresso. (CAMORLINGA, 1997, p. 660). 
Los resultados de nuestro estudio revelan que la actitud hacia la LE cambia considerablemente en esta última etapa respecto a las etapas anteriores. En este grupo el porcentaje de estudiantes que considera el portugués una lengua fácil se mantiene como en la etapa intermedia $(62,5 \%)$, pero se observa que la actitud positiva hacia la semejanza estructural y gramatical entre el portugués y el español se reduce; solamente un 50\% de los encuestados considera que la similitud estructural es una ventaja. Disminuye también la actitud respecto a la semejanza léxica, solo un 37,5\% de los alumnos considera que el parecido del léxico es una ventaja para la traducción. Además, se modifica también la idea que el alumno tenía de la labor de traducción del portugués al español. Ahora solo el 50\% considera que se trata de una tarea fácil. Por otro lado se altera también la percepción del alumno frente a las falsas analogías y la posibilidad de provocar interferencias. A medida que el alumno realiza tareas de traducción, desarrolla una actitud crítica y analiza los problemas que se derivan de la proximidad de estas lenguas, es decir, los alumnos realizan encargos más complejos de traducción, toman conciencia de las dificultades específicas que plantea la traducción de lenguas próximas y la mayoría de ellos considera que, a pesar de poseer una elevada competencia lingüística, el estrecho contacto entre estas lenguas puede provocar transferencias negativas. En la fase final el $50 \%$ de los estudiantes considera que las falsas semejanzas pueden provocar errores de traducción incluso cuando el nivel de conocimiento lingüístico es elevado. En relación a las causas de estos errores, el 87,5\% opina que las falsas analogías provocan interferencias por falta de atención, seguido por el $62,5 \%$ que creen que se debe a desconocimiento del léxico. Por otro lado, aumenta el porcentaje de alumnos que considera que los errores que los falsos amigos originan se deben no solo a las diferencias enmascaradas entre las dos lenguas de trabajo, sino también al contexto en el que aparece el falso amigo (62,5\%), al estado emocional de cada traductor como cansancio o situaciones de estrés (75\%), y a la urgencia y falta de revisión de la traducción $(62,5 \%)$. Aumenta el porcentaje (50\%) de entrevistados que considera la falta de competencia traductora como una causa de error y aumenta también levemente el número de entrevistados $(25 \%)$ que considera un motivo de error la escasez de diccionarios y otras fuentes específicas de documentación. 


\section{Evolución en el proceso de aprendizaje}

Una vez analizados los cuestionarios, hemos comprobado que la actitud del estudiante y su percepción de dificultad o facilidad, así como de distancia o proximidad tipológica entre la LM y la LE varía considerablemente a lo largo de todo el proceso de formación universitaria. Según los resultados de la encuesta realizada, la evolución de la actitud del alumno es la siguiente:

Comparada con la lengua B del estudiante, la lengua portuguesa se considera una lengua fácil a lo largo de todo el proceso de aprendizaje. El 87,5\% de los entrevistados en la fase inicial de adquisición lingüística cree que es una lengua fácil; el 72,7\% considera que el portugués es fácil en la fase intermedia, y el 62,5\% lo cree en la fase de nivel avanzado.

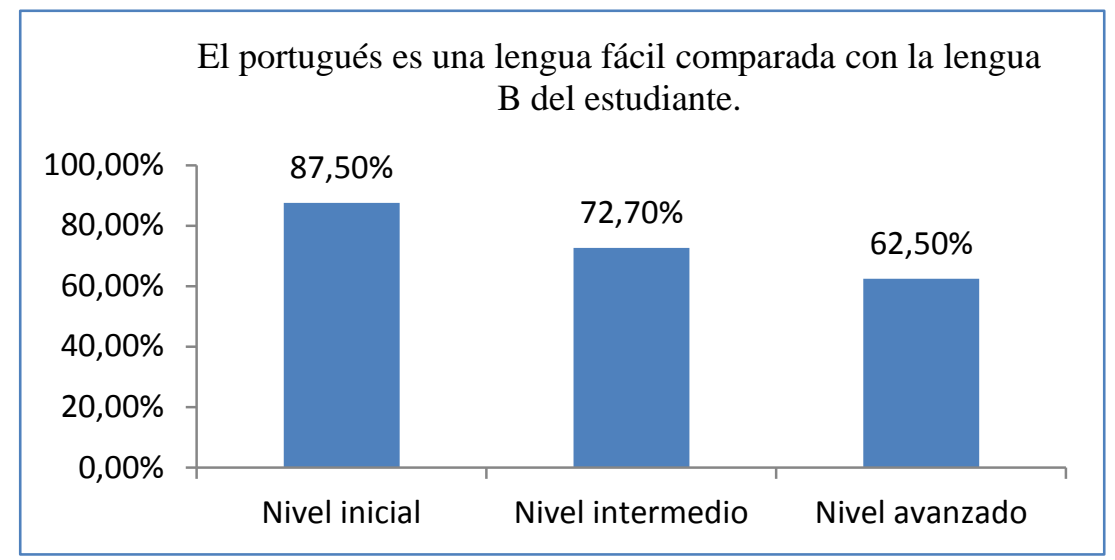

Gráfico 1: Alumnos que están de acuerdo con la siguiente afirmación: El portugués es una lengua fácil comparada con mi lengua $B$.

Se observa, sin embargo, una variación a la hora de considerar la proximidad lingüística como una ventaja respecto a la traducción. En la fase inicial el $75 \%$ de los entrevistados percibe la semejanza estructural y gramatical como una ventaja. Ese porcentaje desciende hasta el $63,63 \%$ en la fase intermedia, y en la etapa de nivel avanzado disminuye hasta el $50 \%$. 


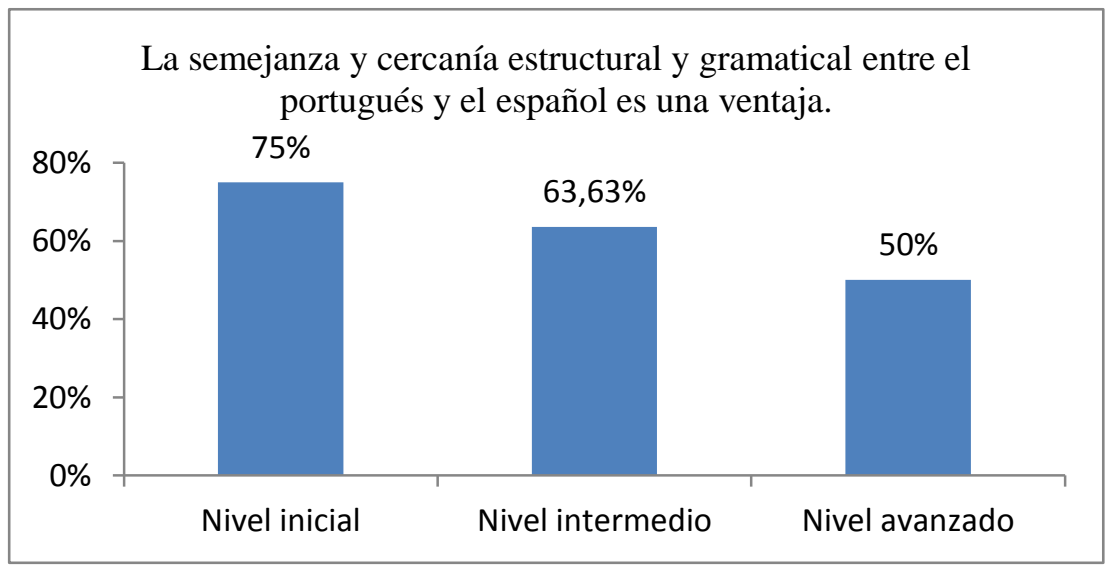

Gráfico 2: Alumnos que están de acuerdo con la siguiente afirmación: La semejanza y cercanía estructural y gramatical entre el portugués y el español es una ventaja.

La confianza que muestra el alumno ante el léxico no es tan alta al inicio de la adquisición de la lengua. Esta situación se produce probablemente porque la mayoría de los manuales de lengua portuguesa hacen hincapié en los falsos amigos semánticos. La proximidad del léxico es una ventaja para el 58,3\% de los entrevistados en la primera fase, en la segunda lo es para el 45,45\% y en la última etapa solo el 37,50\% declara que la similitud léxica es una ventaja. 
La semejanza y cercanía del léxico entre el portugués y el español es una ventaja.

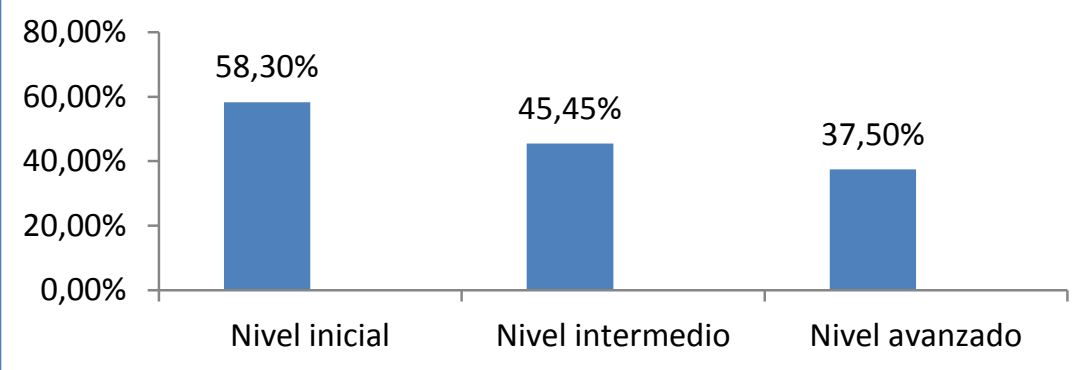

Gráfico 3: Alumnos que están de acuerdo con la siguiente afirmación: La semejanza y cercanía del léxico entre el portugués y el español es una ventaja.

Respecto a la percepción de facilidad o dificultad de traducir del portugués al español, en la primera etapa de aprendizaje el $75 \%$ cree que será una tarea fácil, aunque los alumnos todavía no han realizado ejercicios prácticos de traducción. El 63,6\% lo considera en la segunda fase y paradójicamente al final de su formación universitaria, cuando el alumno posee un buen dominio lingüístico, este porcentaje baja considerablemente y tan solo el $50 \%$ opina que traducir del portugués al español es una labor sencilla. 


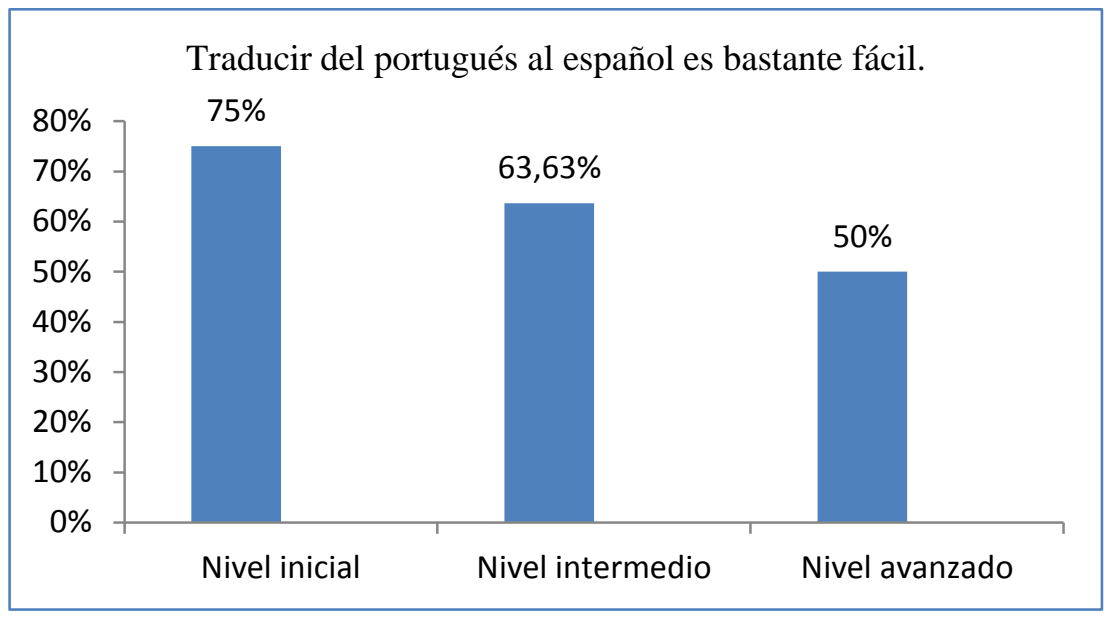

Gráfico 4: Los alumnos están de acuerdo con la siguiente afirmación: Traducir del portugués al español es bastante fácil.

Se observa un cambio de actitud considerable en los diferentes niveles de aprendizaje respecto a la creencia del alumno de posibilidad de interferencia de las falsas analogías. En la primera fase de aprendizaje solo el $25 \%$ cree que las falsas analogías pueden provocar errores de traducción incluso cuando el nivel de competencia lingüística es elevado; el 36,36\% tiene esa opinión en la fase de nivel intermedio, y el 50 en la fase de nivel avanzado. 


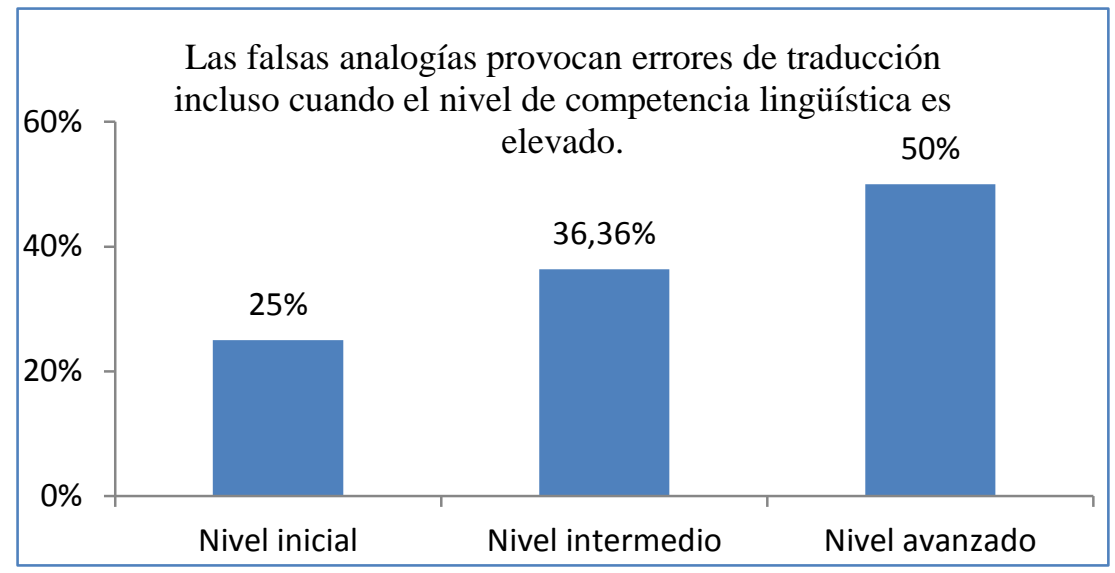

Gráfico 5: Los alumnos están de acuerdo con la siguiente afirmación: Las falsas analogías provocan errores de traducción incluso cuando el nivel de competencia lingüistica es elevado.

En relación a las causas por las que las falsas analogías provocan errores de traducción, la mayoría considera a lo largo de todo el proceso de aprendizaje que el desconocimiento del léxico puede provocar errores e interferencias: el $87,5 \%$, en la fase inicial de aprendizaje, el $72,7 \%$, en la segunda fase y el $62,5 \%$ en la fase de nivel avanzado. Se observa, sin embargo, que a medida que el alumno avanza en su formación, su actitud respecto a la traducción se hace más crítica y, como se observa en el siguiente gráfico, tiene en consideración otros factores como la falta de atención, el cansancio y el estrés o el contexto textual. 


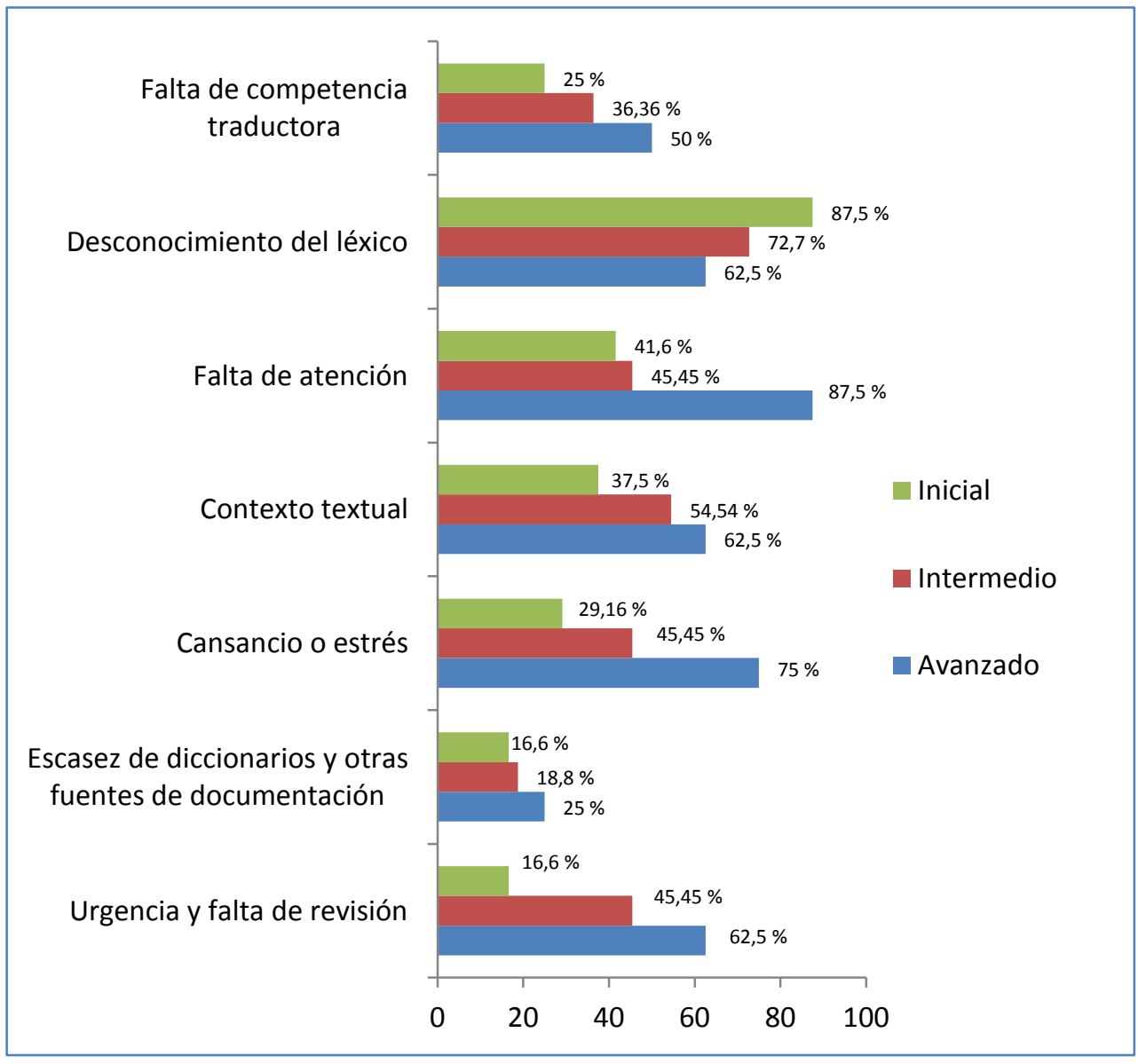

Gráfico 6: Porcentajes de las causas que pueden provocar errores de traducción.

\section{Consideraciones finales}

Analizar la actitud y las ideas preconcebidas del estudiante en el transcurso de su formación permite comprender mejor todo el proceso de aprendizaje y conocer los factores afectivos que inciden en la adquisición de la LE. Este estudio nos muestra que la percepción de 
distancia lingüística que tiene el alumno varía a lo largo del periodo formativo. El alumno tarda en adquirir un conocimiento real de la distancia o proximidad que existe entre la lengua portuguesa y la lengua española y, por consiguiente, tarda en obtener una noción real de la dificultad que entraña la traducción entre estas lenguas. La semejanza lingüística permite avanzar con rapidez en la primera etapa de aprendizaje y crea una actitud marcadamente optimista en el estudiante. Una vez que el alumno empieza a traducir y reflexiona sobre las estrategias que utiliza, comienza a desarrollar una actitud de alerta y toma conciencia de los peligros que esconde esa proximidad. Finalmente, en la fase final de su formación, la práctica de la traducción y el desarrollo de la competencia traductora ajustan la percepción de distancia lingüística entre el portugués y el español.

Como ha quedado demostrado, la actitud del estudiante y su percepción de la lengua extranjera influyen de forma determinante en el proceso de aprendizaje. Cuanto menor es la percepción de distancia, mayor es el grado de confianza y mayor es el riesgo de interferencia. Una actitud crítica y reflexiva contribuye a desarrollar el estrés positivo necesario para mantener el estado de alerta que se precisa para llevar a cabo el proceso de traducción entre estas lenguas. El alumno debe ser consciente del conocimiento real que posee de la lengua portuguesa y conocer la distancia exacta entre el portugués y el español para poder distinguir las semejanzas y las diferencias de las dos lenguas. Para optimizar resultados consideramos que es necesario diseñar una metodología específica para la enseñanza de la lengua portuguesa a hispanohablantes que tenga en cuenta todos los factores inherentes a la proximidad de estas lenguas, no solo los lingüísticos, sino también los factores afectivos como la percepción de dificultad. De este modo se puede rentabilizar al máximo las ventajas de la proximidad lingüística y evitar al mismo tiempo las dificultades asociadas a este par de lenguas.

\section{Referencias}

ALMEIDA FILHO, José Carlos P. Uma metodologia específica para o ensino de línguas próximas? In: (Org.). Português para 
estrangeiros: interface com o espanhol. Campinas: Pontes, 1995. p. 921.

ALONSO REY, Rocío. El mito de la facilidad en el estudio del proceso de enseñanza/aprendizaje de P/LE-HE. Estudios Portugueses: Revista de Filología Portuguesa, n. 4, p. 11-38, 2004.

- La transferencia en el aprendizaje de portugués por hispanohablantes. Salamanca: Luso-Española, 2012.

AMADO ACOSTA, Margarida; ACOSTA, José León. Variedades del español y dialectos idiosincrásicos de los aprendices portugueses. Polifonia, n. 3, p. 11-35, 2000.

AINCIBURU, M. Cecilia. Aspectos del aprendizaje del vocabulario: tipo de palabra, método, contexto y grado de competencia en las lenguas afines. Frankfurt am Main: Peter Lang, 2008.

BAERLOCHER ROCHA, Cynthia; LUCHA CUADROS, R. María. Aportaciones metodológicas y didácticas derivadas de un análisis de errores de ELE en un contexto de lenguas próximas. In: CONTRERAS IZQUIERDO, Narciso M. (Ed.). La enseñanza del español como LE/L2 en el siglo XXI. Jaén, ASELE, 2014. p. 99-109.

BANDURA, Albert. Self-efficacy: the exercise of control. New York: Freeman, 1997.

CALVI, M. Vittoria. Aprendizaje de lenguas afines: español e italiano. redELE: Revista Electrónica de Didáctica ELE, n. 1, 2004. Disponível em: $\quad<$ http://www.mecd.gob.es/dctm/redele/MaterialRedEle/Revista/2004_01/2004_redELE_1_02Calvi.pdf?documentId=0 901e72b80e06a6d > Acesso em: 20 set. 2016.

CALVO CAPILLA, M. Carolina; RIDD, Mark. A tradução como atividade contrastiva e de conscientização na aprendizagem de línguas próximas. Horizontes de Linguística Aplicada, v. 8, n. 2, p. 150-169, 2009.

Disponível em: 
<http://periodicos.unb.br/index.php/horizontesla/article/viewFile/2939/ 2543>. Acesso em: 9 set. 2016.

CAMARENA ORTIZ Eréndira D. Análisis de interlengua en un corpus de portugués escrito de estudiantes castellano hablantes universitarios. Revista de Lingüística y Lenguas Aplicadas, v. 6, p. 7182 2011.

Disponível em: <http://polipapers.upv.es/index.php/rdlyla/article/view/894/964>. Acesso em: 2 set. 2016.

CAMORLINGA, Rafael. A distância da proximidade: a dificuldade de aprender uma língua fácil. Intercâmbio, v. 6, p. 653-671, 1997.

CARLUCCI, Laura; DÍAZ FERRERO, Ana M. Falsas equivalencias en la traducción de lenguas afines: propuesta taxonómica. Sendebar, n. 18, p. 159-191, 2007.

CONSEJO de Europa. Marco común europeo de referencia para las lenguas: aprendizaje, enseñanza, evaluación. Madrid: MECD y Anaya, 2002. Disponível em: <http://cvc.cervantes.es/ensenanza/biblioteca_ele/marco/cvc_mer.pdf> . Acesso em: 26 set. 2016.

DIAS, Eduardo M. De como Eça foi assassinado em Espanha: as primeiras traduções queirosianas. Revista Colóquio/Letras, n. 121/122, p. 131-141, 1991. Disponível em: 〈http://coloquio.gulbenkian.pt>. Acesso em: 9 set. 2016.

DÍAZ FOUCES, Óscar. Algumas considerações sobre a combinação linguística Português-Espanhol. In: (Ed.). Olhares \& miradas: reflexiones sobre la traducción português-español y su didáctica. Granada: Átrio, 2012. p. 119-135.

ELAMANE, Abderrahim. Problemas particulares en la traducción entre parejas de lenguas cercanas y parejas de lenguas de origen diferente. In: CONGRÉS INTERNACIONAL SOBRE TRADUCCIÓ, I, 1992. Barcelona. Actes del I Congrés Internacional sobre Traducció. Bellaterra: Universitat Autónoma de Barcelona, 1996. p. 253-264. 
FERNANDES, Marcia. H. B.; BRITO, Karim S. A psico-tipologia e suas implicações no aprendizado de línguas estrangeiras. Horizontes de Linguística Aplicada, Brasília, v. 2, p. 26-37, 2006.

FÉRRIZ MARTÍNEZ, Carmen; SANS CLIMENT, Carles. Una propuesta de intervención didáctica en la enseñanza de la traducción del portugués al español: análisis de errores de traducción. MarcoELE: Revista de Didáctica, n. 11, p. 37-63, 2010. Disponível em: <http://marcoele.com/descargas/11/03.ferriz_sans.pdf>. Acesso em: 1 de out. 2016.

FRIES, Charles. C. Teaching and learning English as a foreign language. Ann Arbor: The University of Michigan Press, 1945.

GASS, S.; SELINKER, L. (Eds.). Language transfer in language learning. Rowley: Newbury House, 1983.

GÓNZALEZ, Neide T. M. A questão do ensino do espanhol no Brasil. Perspectiva, v. 7, n. 13, p. 74-90, 1989.

. El español para brasileños. Sobre por dónde determinar la justa medida de una cercanía. Anuario Brasileño de Estudios Hispánicos, n. 9, p. 11-19, 1999. Disponível em: <file:///C:/Users/Usuariuo/Downloads/15655.pdf>. Acesso em: 26 set. 2016.

HENRIQUES, Eunice R. Intercompreensão de texto escrito por falantes nativos de português e de espanhol. DELTA, São Paulo, v. 16, n. 2, p. 263-295, 2000.

HUFEISEN, Brita; MARX, Nicole. How can DaFnE and EuroComGerm contribute to the concept of receptive multilingualism? Theoretical and practical considerations. In: THIJE, J. D. ten; ZEEVAERT, L. (Orgs.). Receptive multilingualism: linguistic analysis, language policies and didactic concepts. Amsterdam: John Benjamins, 2007. p. 307-321. 
KULIKOWSKI, M. Zulma Moriondo; GONZÁLEZ KELLERMAN, Eric. Now you see it, now you don't. In: GASS, S. M.; Selinker, L. (Coords.). Language transfer in language learning. Rowley: Newbury House, 1983. p. 112-134.

LADO, Robert. Linguistics across cultures: applied linguistics for language teachers. Ann Arbor: The University of Michigan Press, 1957.

LEIRIA, I. Falemos antes de verdadeiros amigos. In: PINTO, P. F.; JÚDICE N. (Eds.). Para acabar de vez com Tordesilhas. Lisboa: Edições Colibri, 1998. p. 11-29.

LOMBELLO, Leonor C.; EL-DASH, Linda. G.; BALEEIRO, Marisa A. Subsídios para a elaboração de material didático para falantes de espanhol. Trabalhos em Lingüística Aplicada, v. 1, p. 117-132, 1983.

LOZADO, Patrícia Rosa. A aquisição dos pronomes pessoais da Língua Espanhola por falantes de Português do Brasil: aspectos lingüísticos e psicotipológicos. 182 f. Dissertação (Mestrado em Linguística Aplicada) Instituto de Letras, Universidade de Brasília, Brasília, 2007. Disponível em:

<http://repositorio.unb.br/bitstream/10482/3840/1/2007_PatriciaRosaL ozado.pdf> Acesso em: 2 set de 2016.

MÚRIAS, Augusto. Espanhol e português: as dificuldades inesperadas I. A Folha, n. 44, 2014. Disponível em: <http://ec.europa.eu/translation/portuguese/magazine/documents/folha 44_pt.pdf>. Acesso em: 25 set. 2016.

NASCENTES, Antenor. Estudos Filológicos. Rio de Janeiro: Livraria Acadêmica, 1939.

NATEL, Tania B. T. La proximidad entre el portugués y el español ¿facilita o dificulta el aprendizaje? In: CONGRESO INTERNACIONAL DE LA ASOCIACIÓN PARA LA ENSEÑANZA DEL ESPAÑOL COMO LENGUA EXTRANJERA, XIII, 2002. Murcia. El español, lengua del mestizaje y la interculturalidad. 
Actas... Murcia: ASELE, 2002. p. 825-832. Disponível em: <http://cvc.cervantes.es/ensenanza/biblioteca_ele/asele/pdf/13/13_082 5.pdf>. Acesso em: 20 set. 2016.

ODLIN, Terence. Language transfer. Cambridge: Cambridge University Press, 1989.

OLLER, J. W.; ZIAHOSSEINY, S. M. The contrastive analysis hypothesis and spelling errors. Language Learning, v. 20, p. 183-189, 1970.

ORTIZ ÁLVAREZ, M. Luisa. Línguas próximas e interlíngua. In: . (Org.). Novas línguas/línguas novas: questões da interlíngua na pesquisa em linguística aplicada. Campinas: Pontes Editores, 2012. p. 245-264.

PÉREZ PÉREZ, Noemí. La enseñanza de español a lusohablantes portugueses: ventajas e inconvenientes. In: CONGRESO INTERNACIONAL DE LA ASOCIACIÓN EUROPEA DE PROFESORES DE ESPAÑOL, XLII, 2007, Santander. Actas... Madrid: AEPE, 2008. p. 27-35.

PONCE DE LEÓN, Rogelio. Focos y tópicos en contraste: consideraciones sobre la adquisición de procedimientos de focalización y topicalización en alumnos de español lusohablantes. À Beira, n. 6, p. 55-72, 2007. Disponível em: <https://sigarra.up.pt/flup/pt//pub_geral.show_file?pi_gdoc_id=443063 >. Acesso em: 1 out. 2016.

SABIO PINILLA, José Antonio. Traducir del portugués al español: la engañosa facilidad. In: CONGRÉS INTERNACIONAL SOBRE TRADUCCIÓN, I, 1992. Barcelona. Actes. Bellaterra: Universitat Autónoma de Barcelona, 1996. p. 613-620.

ULSH, Jack L. From Spanish to Portuguese. Washington, D. C.: Foreign Service Institute, 1971. 
VILAR SÁNCHEZ, Karin. ¿Imposible el alemán? Cercanía percibida entre lenguas y disposición para el aprendizaje. Lengua y Migración, n. 6, v. 2, p. 67-94, 2014.

VILLANUEVA, Ana M. Efectos de la distancia tipológica entre las lenguas y del número de lenguas conocidas en la adquisición de la sintaxis del español como lengua extranjera. Núcleo, v. 23, n. 28, p. 217-251, 2011. Disponível em: 〈http://www.scielo.org.ve/pdf/nu/v23n28/art09.pdf〉. Acesso em: 1 set. 2016.

WEINREICH, U. Languages in contact: findings and problems. The Hague: Mouton, 1953.

Recebido em: 21/11/2016

Aceito em: 24/05/2017

Título: Percepção da distância linguística na aquisição da língua portuguesa por hispanofalantes 